\title{
Magnetic properties of graphitically encapsulated nickel nanocrystals
}

\author{
J.-H. Hwang and V.P. Dravid \\ Department of Materials Science and Engineering, Materials Research Center, Northwestern University, \\ Evanston, Illinois 60208
}

M. H. Teng

Department of Geology, National Taiwan University, Taipei, Taiwan, Republic of China

\author{
J. J. Host, B. R. Elliott, D. L. Johnson, and T. O. Mason \\ Department of Materials Science and Engineering, Materials Research Center, Northwestern University, \\ Evanston, Illinois 60208
}

(Received 8 July 1996; accepted 23 December 1996)

\begin{abstract}
Graphitically encapsulated ferromagnetic Ni nanocrystals have been synthesized via a modified tungsten arc-discharge method. By virtue of the protective graphitic coating, these nanocrystals are stable against environmental degradation, including extended exposure to strong acids. The magnetic properties of the encapsulated particles are characterized with regard to the nanoscale nature of the particles and the influence of the graphitic coating which is believed to be benign insofar as the intrinsic magnetic properties of the encapsulated nanocrystals are concerned. The Curie temperature of graphitically encapsulated $\mathrm{Ni}$ nanocrystals is the same as that of microcrystalline Ni. However, saturation magnetization, remanent magnetization, and coercivity of these particles are reduced, for a range of temperatures. The unique features are compared with those of unencapsulated nanocrystalline and coarse microcrystalline nickel particles.
\end{abstract}

\section{INTRODUCTION}

Nanocrystalline materials with particle or grain sizes in the range $10-100 \mathrm{~nm}$ have gained considerable attention in recent years because of the potential for enhanced or even unique physical properties compared to their microcrystalline or bulk counterparts. ${ }^{1-5}$ A variety of metals and nonmetallic materials have been synthesized by gas phase condensation ${ }^{6-12}$ and aqueous chemical routes. ${ }^{13-15}$ Nanocrystalline metals, although readily available, have the disadvantage of being prone to rapid environmental degradation, owing to a very high surface area to volume ratio and high reactivity. This tends to limit potential industrial applications and even scientific evaluation of nanocrystalline properties. A recent breakthrough in this regard is encapsulation of nanocrystals with chemically stable species such as graphitic layers,${ }^{16-29}$ which not only protects the nanocrystals from environmental degradation, but is also believed to be benign insofar as the intrinsic nanocrystalline magnetic properties are concerned.

The recent studies of the graphitic encapsulation of metal carbides ${ }^{16-23}$ have promoted an extensive research on similar protection of ferromagnetic materials, e.g., $\mathrm{Fe}, \mathrm{Co}$, and $\mathrm{Ni}^{24-29}$ Appropriately encapsulated ferromagnetic materials may find applications ranging from ferrofluids and recording media to novel biomedical applications such as drug delivery and immunoassays.
Such potential applications pose stringent requirements of the physical and magnetic properties of nanocrystals. The synthesis method employed should result in high quality encapsulated nanocrystals of well-defined and controlled size distribution with high yield. In addition, carbonaceous debris should be minimal and the graphitic coating should be clean and amenable to surface chemical treatment. Unfortunately, the conventional graphitegraphite arc method utilized to synthesize graphitically encapsulated ferromagnetic nanocrystals suffers from many shortcomings. The conventional process provides little or no control over the particle size, the yield is often low, and there is considerable carbonaceous debris which is very difficult to remove from the desired product. Despite these difficulties, a number of researchers have made significant headway in the synthesis and characterization of graphitically encapsulated ferromagnetic nanocrystals and metal carbides. ${ }^{16-29}$ McHenry et al. ${ }^{24,28}$ reported carbon encapsulation of ferromagnetic metals, and conducted magnetic property measurements. They showed that encapsulated ferromagnetic Co nanocrystals dispersed in epoxy exhibited superparamagnetic behavior at room temperature. ${ }^{28}$ Paramagnetic behavior was observed in encapsulated $\mathrm{Gd}_{2} \mathrm{C}_{3}$ nanocrystals. ${ }^{22}$ Encapsulation of $\mathrm{Fe}$ (including $\alpha-\mathrm{Fe}, \gamma-\mathrm{Fe}$, and $\mathrm{Fe}_{3} \mathrm{C}$ ) produced larger coercivity than is seen in microcrystalline materials. ${ }^{29}$ However, encapsulated $\mathrm{TaC}$ exhibited 
the same superconducting transition temperature, $T_{c}$, as the bulk materials, and antiferromagnetic nanocrystalline $\mathrm{CeC}_{2}$ was reported to have the same Néel temperature as bulk specimens. ${ }^{23}$

As an alternative to the graphite-graphite arc process, we have recently been successful in encapsulating significant quantities of ferromagnetic nanocrystals $(\mathrm{Ni}, \mathrm{Co}$, and $\mathrm{Fe})$ in graphitic shells using a modified $\mathrm{W}$-arc method. ${ }^{30}$

In this study, the magnetic properties of graphitically encapsulated $\mathrm{Ni}$ nanocrystals are evaluated, including the Curie temperature, and magnetization versus magnetic field (M-H plots) as a function of temperature. The magnetic properties of graphitically encapsulated $\mathrm{Ni}$ nanocrystals are then compared with those of large grain size nickel particles and with values reported in the literature for nanocrystalline and amorphous nickel.

\section{EXPERIMENTAL PROCEDURE}

The modified tungsten arc apparatus and synthesis procedure have been fully described elsewhere. ${ }^{31} \mathrm{~A}$ tungsten rod was used as a cathode while a molten $\mathrm{Ni}$ pool supported by a graphite crucible was employed as the anode of the material to be encapsulated. An arc was generated at a helium pressure of 400 Torr at $130 \mathrm{~A}$ and 20-21 V. The arc plasma produced a mixture of bare nanocrystalline $\mathrm{Ni}$, Ni particles encapsulated in graphitic shells, and some carbonaceous debris. The resultant powder was leached in strong acids, e.g., hydrochloric acid, nitric acid, or aqua regia to dissolve unencapsulated nanocrystals. Subsequently, the encapsulated Ni component of the powder was separated with a strong magnet from the solution, washed, and dried under ambient conditions. Magnetic separation removes a portion of carbonaceous debris from the encapsulated Ni powder.

The encapsulation of nickel particles was confirmed by a field emission transmission electron microscope (FE TEM, HF-2000, Hitachi Ltd., Tokyo, Japan). The particle size distribution and average particle size were determined using TEM micrographs. The phase was identified by x-ray diffractometer (XDS 2000, Scintag Inc., Santa Clara, CA). The encapsulated Ni powder was pressed into a disk (1/8 in. in diameter) at $200 \mathrm{MPa}$. Microcrystalline $(2-3 \mu \mathrm{m}) \mathrm{Ni}$ powder was similarly pressed into a disk to provide a reference specimen. Magnetic measurements on compacted specimens were made using a SQUID magnetometer (MPMS, Quantum Design, Inc., San Diego, CA) at $10 \mathrm{~K}$ and $300 \mathrm{~K}$ with a magnetic field up to $3 \times 10^{4} \mathrm{Oe}$. The weight ratio of $\mathrm{Ni}$ and $\mathrm{C}$ and thermal stability were studied through a subsequent oxidation experiment and thermogravimetric analysis (DuPont 951 Thermogravimetric Analyzer, DuPont Company, Wilmington, DE).

\section{RESULTS AND DISCUSSION}

An x-ray diffraction (XRD) scan of the graphitically encapsulated Ni particles is shown in Fig. 1, indicating the sole presence of fcc nickel. The broadening of the diffraction peaks is attributed to the small particle size. Diffraction peaks of graphite were not observed, indicating the nanoscale nature and/or low content of graphitic layers. Of course, any amorphous carbonaceous debris would not be detected. The presence of graphitic encapsulation was identified by HREM lattice imaging (Fig. 2), nanodiffraction and x-ray microanalysis. ${ }^{32}$ The particle size distribution is shown in Fig. 3; the average particle size was $18.4 \pm 9.2 \mathrm{~nm}$. It should be noted that the nickel particles were protected from aggressive attack by strong acids, by virtue of the graphitic layers.

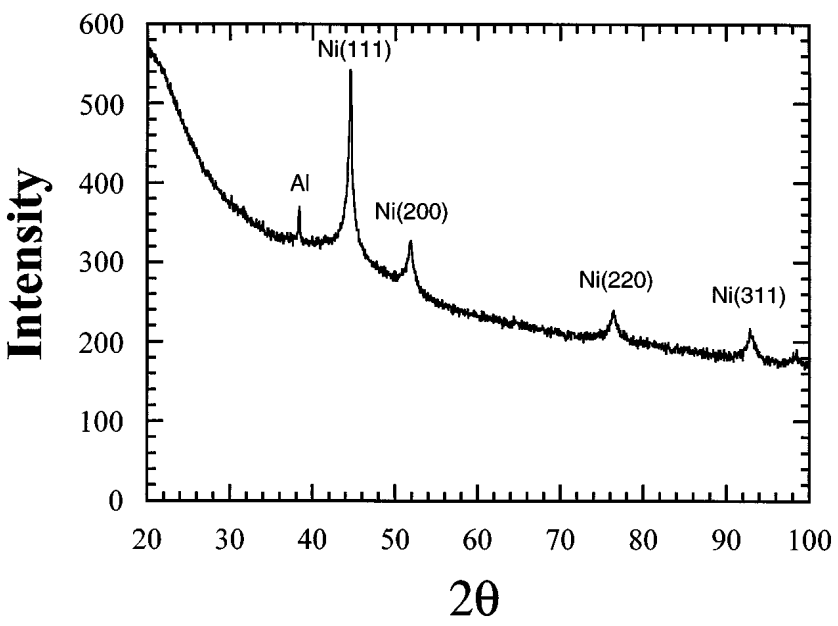

FIG. 1. X-ray diffraction pattern of graphitically encapsulated Ni nanocrystals. (The aluminum peak is due to the $\mathrm{x}$-ray specimen holder.)

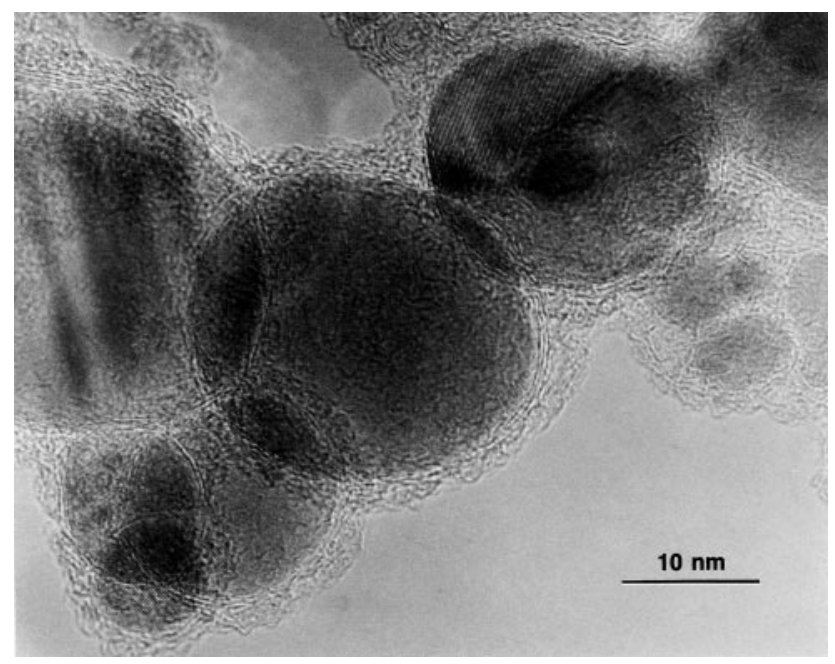

FIG. 2. HREM micrograph of nickel particles encapsulated with several graphitic layers. 


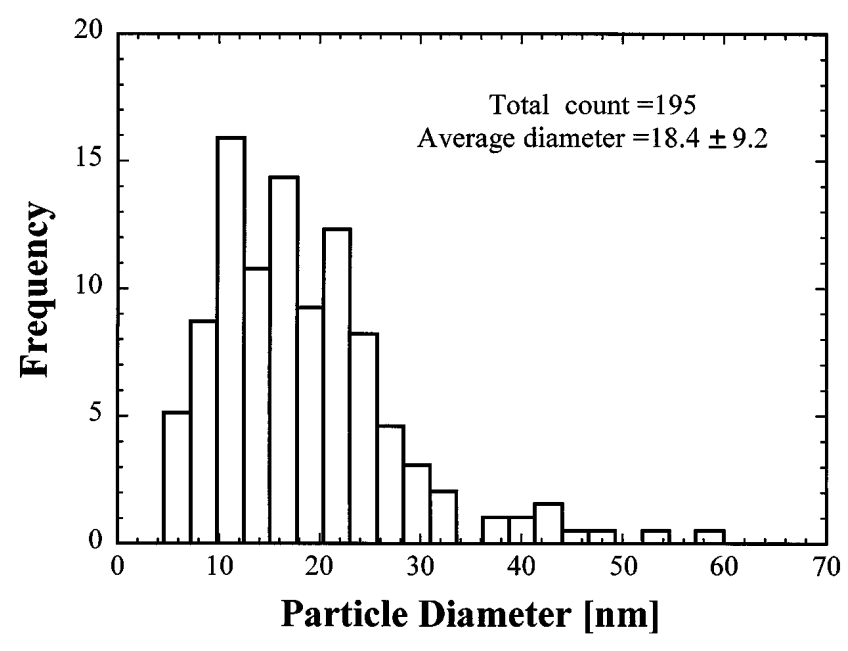

FIG. 3. Particle size distribution histogram of graphitically encapsulated nickel particles. The thickness of graphitic layers was not considered.

The difference in weight before and after high temperature treatment in air allows the relative weight ratio of carbon to nickel to be calculated. At room temperature, the initial state consists of $\mathrm{Ni}$ and carbon, while the final product is made up of nickel oxide, after $\mathrm{CO}_{2}$ formation and thermal oxidation of $\mathrm{Ni}$ at high temperature. Therefore, the weight of nickel oxide can be used to calculate the carbon amount from the initial weight. In this work, the weight percentages of $\mathrm{Ni}$ and $\mathrm{C}$ were estimated from two thermal oxidation experiments. One was performed in a furnace at $900{ }^{\circ} \mathrm{C}$ for $12 \mathrm{~h}$ and the other was performed under flowing air atmosphere at $900{ }^{\circ} \mathrm{C}$ for $6 \mathrm{~h}$. The average weight percentages of $\mathrm{Ni}$ and $\mathrm{C}$ are calculated to be $60.9 \%$ and $39.1 \%$, respectively, with an approximate experimental error of $9.2 \%$. Considering the number of the graphitic layers with regard to the average particle size, the estimated weight of $\mathrm{C}$ is less than the current value, indicating the presence of approximately $33 \%$ carbonaceous debris. ${ }^{32}$ From thermogravimetric analysis, the encapsulation shells appear to be stable below $\sim 200{ }^{\circ} \mathrm{C}$ in air. Above that temperature, the conversion from $\mathrm{C}$ to $\mathrm{CO}_{2}$ and/or $\mathrm{CO}$ and the subsequent oxidation of $\mathrm{Ni}$ takes place. Under vacuum, the encapsulated particles are stable to still higher temperatures.

The magnetization versus field plots (i.e., $\mathrm{M}-\mathrm{H}$ loops) for graphitically encapsulated $\mathrm{Ni}$ nanocrystals are shown in Fig. 4 (at $300 \mathrm{~K}$ ) and Fig. 5 (at $10 \mathrm{~K}$ ). At $300 \mathrm{~K}$, the saturation magnetization reaches $\sim 79 \%$ of bulk $\mathrm{Ni}$ with regard to the $\mathrm{Ni}$ content of the specimen, whereas remanent magnetization and coercivity decrease to $\sim 32 \%$ and $\sim 15 \%$ of that of microcrystalline $\mathrm{Ni}$, respectively. The detailed information is listed in Table I.

Figure 6 shows a plot of the magnetization versus temperature (i.e., Curie plot) for graphitically en-
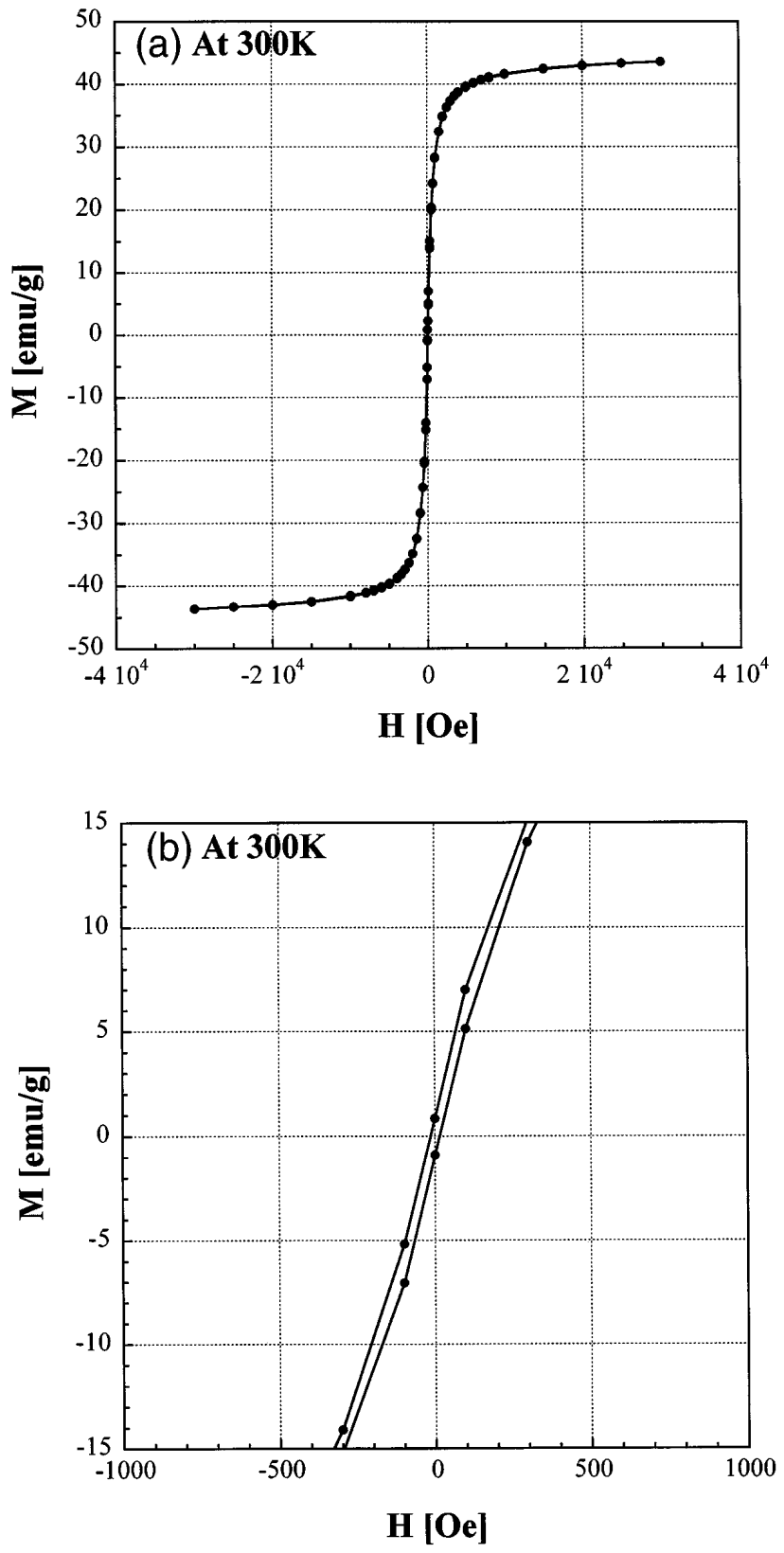

FIG. 4. (a) Magnetization versus magnetic field plot for graphitically encapsulated nickel nanocrystals at $300 \mathrm{~K}$. (b) Enlargement of magnetization versus magnetic field near the origin.

capsulated $\mathrm{Ni}$ nanocrystals kept under vacuum. The extrapolated Curie temperature was determined to be $358 \pm 10{ }^{\circ} \mathrm{C}$. This value is in agreement with the $T_{c}$ of microcrystalline $\mathrm{Ni}^{33}$ However, Du et al. claimed that the $T_{c}$ of nanocrystalline $\mathrm{Ni}$ decreased with decreasing crystal size. ${ }^{34}$ The evidence for this is not clear from the published magnetization versus temperature curve. ${ }^{34}$ McHenry et al. ${ }^{24}$ reported a similar trend in encapsulated Ni nanocrystals prepared using the Kratschmer arc process. 

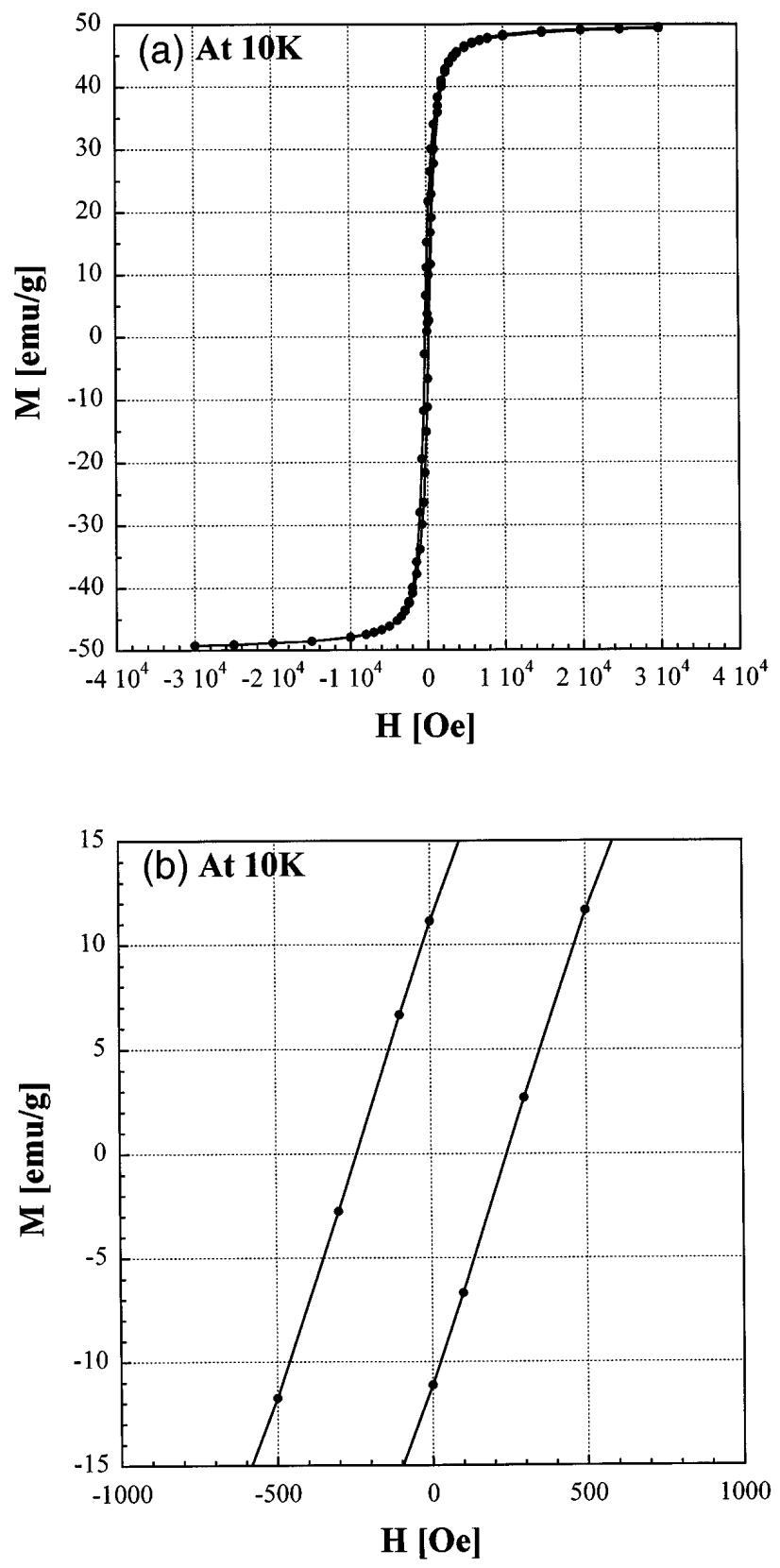

FIG. 5. (a) Magnetization versus magnetic field plot in graphitically encapsulated nickel nanocrystals at $10 \mathrm{~K}$. (b) Enlargement of magnetization versus magnetic field near the origin.
The saturation magnetization of encapsulated $\mathrm{Ni}$ nanocrystals is suppressed in magnitude compared to that of the microcrystalline Ni, as shown in Table I. Near the Curie temperature, the curvature of the magnetization is smaller than that of the bulk $\mathrm{Ni}$; the magnetization decreases gradually toward the Curie temperature. A similar phenomenon was observed in unencapsulated $\mathrm{Ni}$ nanocrystals. ${ }^{34}$ No oxides or nickel carbides were detected. However, a small amount of carbon solubility in these nickel nanocrystals cannot be discounted, which may also reduce magnetization compared to purer constituents. Therefore, the gradual decrease in saturation magnetization can be attributed to the nanocrystalline nature of the encapsulated particles, coupled with possible carbon solution in nickel nanocrystals.

Graphitically encapsulated Ni nanocrystals, with an average size of about $18.2 \mathrm{~nm}$, may be considered to have just a single magnetic domain, the origin of magnetic hysteresis being spin rotation. The magnetic behavior is therefore size-dependent and should be understood in conjunction with thermal energy and surface anisotropy aspects. In particular, single domain particles below a certain critical diameter are greatly affected by temperature. For graphitically encapsulated $\mathrm{Ni}$ nanocrystals, the room temperature remanent magnetization and magnetic coercivity are greatly reduced compared to their coarse polycrystal counterparts. The reduced remanent magnetization and coercivity at room temperature is close to one of the characteristics of superparamagnetism.

Compared to microcrystalline nickel particles, the significant decrease in $M_{r}$ and $H_{c}$ at $300 \mathrm{~K}$ of graphitically encapsulated $\mathrm{Ni}$ nanocrystals implies that the thermal energy to demagnetize becomes dominant over spontaneous magnetization. At $10 \mathrm{~K}$ (see Fig. 5), graphitically encapsulated nickel particles show much larger remanent magnetization and coercivity, as a result of the significant decrease in thermal energy, compared with $300 \mathrm{~K}$. The above trend suggests that the magnetic properties of graphitically encapsulated Ni nanocrystals are controlled by the particle size and can exhibit characteristics associated with superparamagnetism below a critical diameter smaller than in this study.

Amorphous nickel also exhibits a decrease in $M_{s}$ and $T_{c}$, compared with microcrystalline nickel. ${ }^{35}$ A SANS

TABLE I. Comparison of saturation magnetization $\left(M_{s}\right)$, remanent magnetization $\left(M_{r}\right)$, and coercivity $\left(H_{c}\right)$ in encapsulated nanocrystalline and bulk $\mathrm{Ni}$.

\begin{tabular}{|c|c|c|c|c|c|}
\hline & $T(\mathrm{~K})$ & $M_{s}(\mathrm{emu} / \mathrm{g})$ & $M_{r}(\mathrm{emu} / \mathrm{g})$ & $H_{c}(\mathrm{Oe})$ & $d$ \\
\hline Graphitically encapsulated Ni nanocrystals & 300 & $43.7 \pm 4.0$ & $0.86 \pm 0.08$ & 15 & $18.2 \pm 9.2 \mathrm{~nm}$ \\
\hline Bulk Ni & 300 & 55 & 2.7 & 100 & $2-3 \mu \mathrm{m}$ \\
\hline Graphitically encapsulated Ni nanocrystals & 10 & $49.5 \pm 4.6$ & $11.2 \pm 1.0$ & 250 & $18.2 \pm 9.2 \mathrm{~nm}$ \\
\hline
\end{tabular}




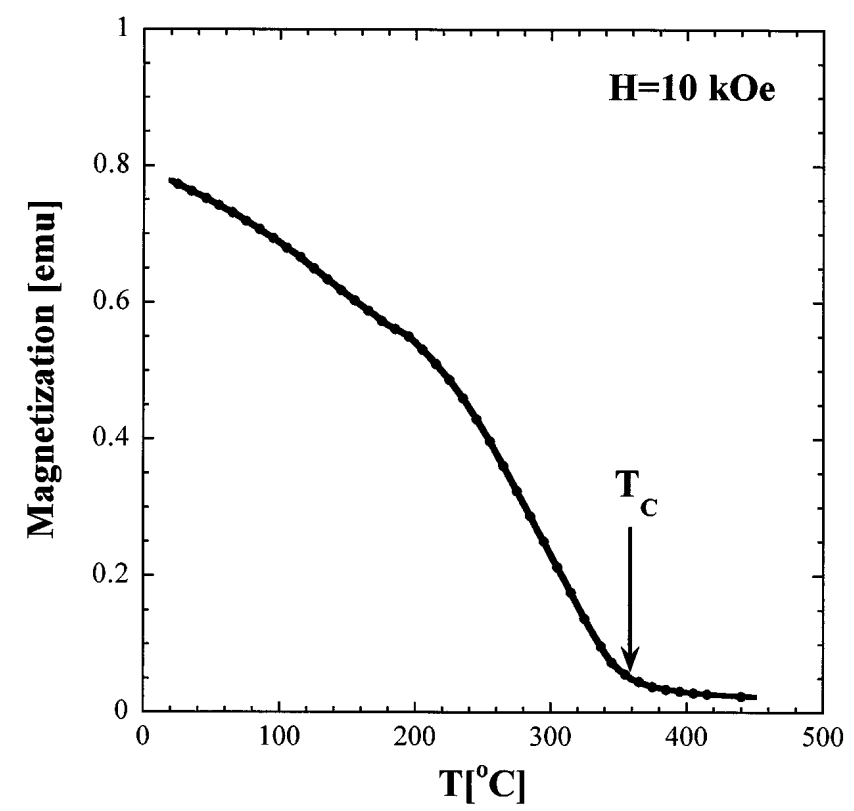

FIG. 6. Magnetization versus temperature of graphitically encapsulated $\mathrm{Ni}$ nanocrystals. (The data were taken under vacuum with a magnetic field of $10 \mathrm{kOe}$.)

(small angle neutron scattering) study on nanocrystalline $\mathrm{Fe}^{36}$ concluded that nanocrystalline $\mathrm{Fe}$ consists of ferromagnetic cores of $\mathrm{Fe}$ and nonmagnetic or less magnetic interfaces. In this context, the term "interface" denotes an interruption of lattice periodicity, such as that found at a grain boundary or a surface. The above reports ${ }^{35,36}$ indicate that the disordered structure in amorphous material and at interfaces provide less magnetic moment per unit mass than that of ferromagnetic core regions. From the temperature dependence of $M_{s}$ in unencapsulated $\mathrm{Ni}$ particles, ${ }^{37}$ the reduced magnetic moment at the interfacial regions was calculated. The detrimental effect of interfaces on saturation magnetization, $M_{s}$, increases dramatically with decreasing particle sizes since the volume fraction of interface increases. Consequently, there is a good probability that the decrease in $M_{s}$ for graphitically encapsulated Ni nanocrystals is due to the nonmagnetic or weakly magnetic interfaces which lead to a decrease in the effective magnetic moment per unit mass. The other possibility is that some carbon may have remained trapped inside $\mathrm{Ni}$ nanocrystals as metastable solid solution. The effect of graphite on the magnetization behavior of encapsulated nanocrystals was also considered. Graphite is diamagnetic, with $21.50 \times$ $10^{-6} \mathrm{emu} / \mathrm{g}$ parallel to magnetic field and $-0.5 \times$ $10^{-6} \mathrm{emu} / \mathrm{g}$ perpendicular to the magnetic field. ${ }^{38}$ Since the mass susceptibility of nickel is much higher than that of graphite, the effect of graphitic and amorphous layers is likely to be very small.

The saturation magnetization, $M_{s}$, and magnetic coercivity, $H_{c}$, of graphitically encapsulated Ni nanocrys- tals as a function of grain size are compared with those of bare nickel nanocrystals, ${ }^{20,37,39-41}$ in Fig. 7 and Fig. 8, respectively. The magnetic saturation and coercivity values are significantly scattered, without any particular trend. Aus et al. ${ }^{41}$ reported that the saturation magnetization is independent of particle size and that an electroplated $\mathrm{Ni}$ film can exclude the effect of an oxide layer. Except in Aus et al., ${ }^{41}$ unencapsulated Ni particles were synthesized using gas evaporation or sputtering and the materials were subsequently exposed to ambient conditions. The formation of oxide layers cannot be avoided due to the thermodynamic instability of nanocrystalline metals like Ni. Secondary phases were reported in ultrafine nickel powders: $\mathrm{NiO}, \mathrm{Ni}_{2} \mathrm{O}_{3}$, and $\mathrm{Ni}(\mathrm{OH})_{2} \cdot 34,37,42,43$ Schaefer et al., Gong et al., and $\mathrm{Du}$ et al. ${ }^{34,37,40}$ suggested the possibility that the oxidation layer induces exchange coupling between ferromagnetic $\mathrm{Ni}$ and antiferromagnetic $\mathrm{NiO}$, retaining high coercivity. As can be seen in Fig. 8, unencapsulated nickel nanocrystals that were exposed to ambient conditions show significant deviations, making the postulate difficult to validate. Interestingly, our encapsulated nickel nanocrystals exhibited much lower $H_{c}$ than the exposed nanocrystalline nickels. Considering that the graphitic encapsulation tends to protect the nanocrystals from any environmental degradation, and that the nanocrystals are pure $\mathrm{Ni}$ metal discounting carbon solid solution, the reduction of $H_{c}$ appears to be intrinsic to the nanocrystals owing to their small size, and the contribution from high density of surface atoms with respect to the bulk.

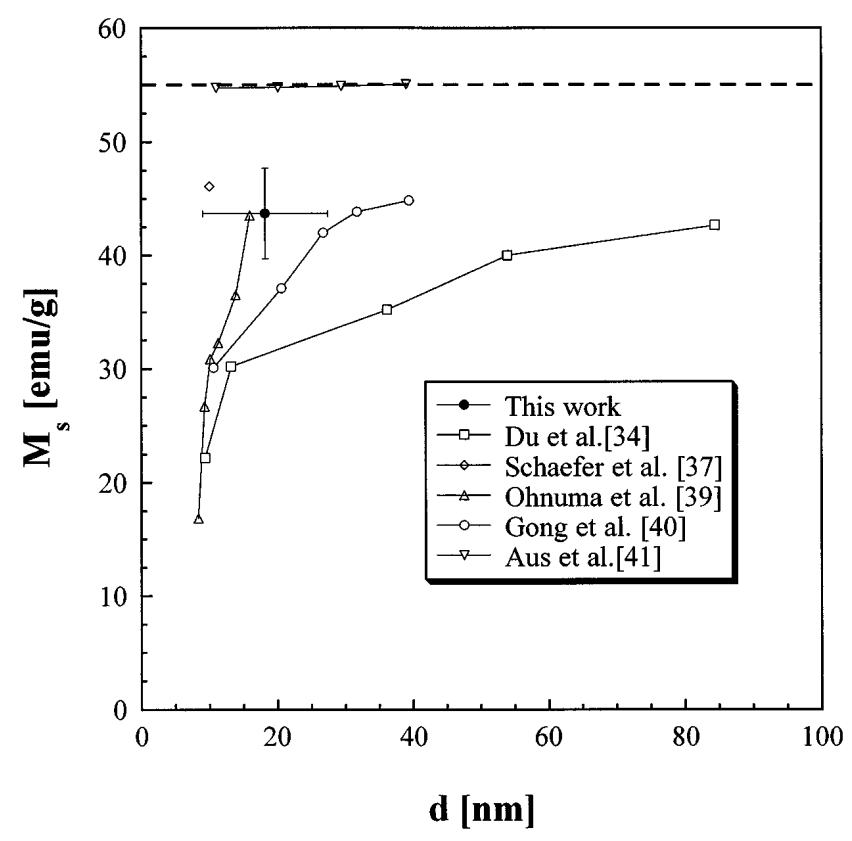

FIG. 7. Comparison of magnetic saturation $\left(M_{s}\right)$ in encapsulated nanocrystalline nickel particles and unencapsulated nickel particles. (The dashed line indicates the measured value from microcrystalline Ni.) 


\section{CONCLUSION}

Graphitically encapsulated nanocrystalline Ni particles were synthesized using an arc-discharge technique, and their various magnetic properties were measured. The Curie temperature of encapsulated Ni nanocrystals is in general agreement with that of the bulk and unencapsulated nanocrystals. However, magnetization with temperature is seen to be suppressed compared to that of bulk nickel. Generally, the encapsulated Ni nanocrystals show significant decreases in magnetic coercivity and saturation magnetization at room temperature, compared with those of bulk Ni. However, the difference seems to be associated with the significant increase in surface area due to the nanoscale particle size and unlikely due to the graphitic coating. However, possible minute carbon solubility cannot be ruled out at this time.

The obvious environmental and thermal stability of graphitically encapsulated nanocrystals, coupled with retention of their intrinsic magnetic properties provide interesting opportunities to develop their technological applications. The lack of coarsening of encapsulated nanocrystals (owing to the blocking graphitic sheets) and any other degradation (e.g. oxidation) with temperature also opens opportunities to investigate the temperature dependence of nanocrystal properties which is not otherwise possible.

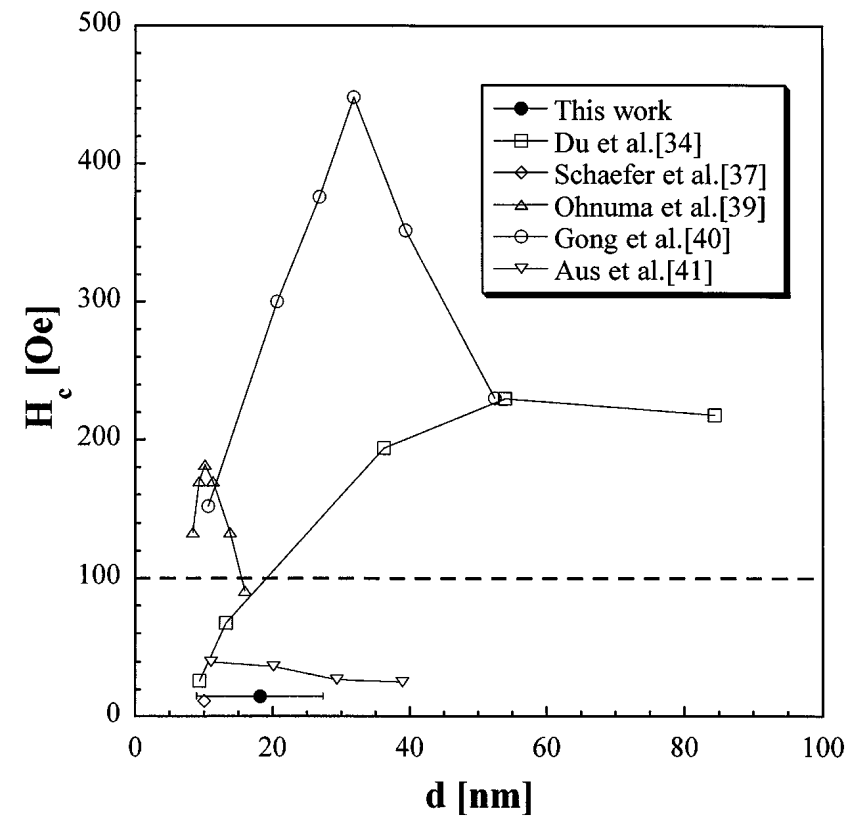

FIG. 8. Comparison of magnetic coercivity $\left(H_{c}\right)$ in encapsulated nanocrystalline nickel particles and unencapsulated nickel particles. (The dashed line indicates the measured value from microcrystalline Ni.)

\section{ACKNOWLEDGMENTS}

This work was supported by National Science Foundation, Grant DMR-9202574, and by the MRL program of the National Science Foundation, at the Materials Research Center of Northwestern University, under Award No. DMR-9120521. VPD is also supported by the NSFNYI program (DMR-9357513), with matching support from DuPont Co. and Exxon Education Foundation. The authors wish to thank M.Z. Lin for the technical assistance in SQUID measurement.

\section{REFERENCES}

1. J. Karch, R. Birringer, and H. Gleiter, Nature (London) 330, 556 (1987).

2. H. Gleiter, Prog. Mater. Sci. 33, 223 (1989).

3. H. Hahn and R. S. Averback, J. Am. Ceram. Soc. 74 (11), 2918 (1991).

4. D. D. Beck and R. W. Siegel, J. Mater. Res. 7, 2840 (1992).

5. R. D. McMichael, R. D. Shull, L. J. Swartzendruber, and L.H. Bennett, J. Magn. Magn. Mater. 11, 29 (1992).

6. C. G. Granqvist and R. A. Buhrman, J. Appl. Phys. 47 (5), 2200 (1976).

7. R. Birringer, H. Gleiter, H. P. Klein, and P. Marquardt, Phys. Lett. 102A (8), 365 (1984).

8. G. W. Nieman, J. R. Weertman, and R. W. Siegel, J. Mater. Res. 6, 1012 (1991).

9. G. E. Fougee, J. R. Weertman, and R. W. Siegel, Nanostructured Mater. 5 (2), 127 (1995).

10. R. W. Siegel, S. Ramasamy, H. Hahn, L. Zongquan, L. Ting, and R. Gronsky, J. Mater. Res. 3, 1367 (1988).

11. J. Lee, J.-H. Hwang, J. J. Mashek, T. O. Mason, A. E. Miller, and R. W. Siegel, J. Mater. Res. 10, 2295 (1995).

12. G. Skandan, Nanostructured Mater. 5 (2), 111 (1995).

13. Y. C. Zhou and M. N. Rahaman, J. Mater. Res. 8, 1680 (1993).

14. S. E. Bates, W. E. Buhro, C. A. Frey, S. M. L. Sastry, and K. F. Kelton, J. Mater. Res. 10, 2599 (1995).

15. D. W. Matson, J. C. Linehan, and M. E. Geusic, Part. Sci. Technol. 10, 143 (1994).

16. R. S. Ruoff, D. C. Lorents, B. Chen, R. Malhotra, and S. Subramoney, Science 259, 346 (1993).

17. B. Diggs, A. Zhou, C. Silva, S. Kirkpatrick, N. T. Nuhfer, M. E. McHenry, D. Petasis, S. A. Majetich, B. Brunett, J.O. Artman, and S. W. Staley, J. Appl. Phys. 75 (10), 5879 (1994).

18. Y. Saito, T. Yoshikawa, M. Okuda, M. Ohkohchi, Y. Ando, A. Kasuya, and Y. Nishina, Chem. Phys. Lett. 209, 72 (1993).

19. M. Tomita, Y. Saito, and T. Hayashi, Jpn. J. Appl. Phys. 32, L280 (1993).

20. Y. Saito, M. Okuda, T. Yoshikawa, A. Kasuya, and Y. Nishina, J. Phys. Chem. 98, 6696 (1994).

21. S. Subramoney, R. S. Ruoff, D. C. Lorents, B. Chan, R. Malhotra, M. J. Dyer, and K. Parvin, Carbon 32 (3), 507 (1994).

22. S. A. Majetich, J. O. Artman, M.E. McHenry, N. T. Nuhfer, and S. W. Staley, Phys. Rev. B 48 (22), 16845 (1993).

23. Y. Murakami, T. Shibata, K. Okuyama, T. Arai, H. Suematsu, and Y. Yoshida, J. Phys. Chem. Solids 54 (12), 1861 (1993).

24. M. E. McHenry, Y. Nakamura, S. Kirkpatrick, F. Johnson, S. Curtin, M. De Graef, and N. T. Nuhfer, in Proc. of the Symposium on Recent Advances in the Chemistry and Physics of Fullerenes and Related Materials, edited by K. M. Kadish and R. S. Ruoff (The Electrochemical Society, Inc., Pennington, NJ, 1994), p. 1463. 
25. Y. Saito, T. Yoshikawa, M. Okuda, N. Fujimoto, K. Sumiyama, K. Suzuki, A. Kasuya, and Y. Nishina, J. Phys. Chem. Solids 54 (12), 1849 (1993).

26. Y. Saito, T. Yoshikawa, M. Okuda, N. Fujimoto, S. Yamamuro, K. Wakoh, K. Sumiyama, K. Suzuki, A. Kasuya, and Y. Nishina, J. Phys. Chem. Solids 75 (1), 134 (1994).

27. E. M. Brunsman, R. Sutton, E. Bortz, K. Kirkpatrick, K. Midelfort, J. Williams, P. Smith, M.E. McHenry, S. A. Majetich, J.O. Artman, M. De Graef, and S. W. Staley, J. Appl. Phys. 75 (10), 5882 (1994).

28. M. E. McHenry, S. A. Majetich, J. O. Artman, M. De Graef, and S. W. Staley, Phys. Rev. B 49 (16), 11358 (1994).

29. T. Hihara, H. Onodera, K. Sumiyama, K. Suzuki, A. Kasuya, Y. Nishina, Y. Saito, T. Yoshikawa, and M. Okuda, Jpn. J. Appl. Phys. 33, L24 (1994).

30. V.P. Dravid, J. J. Host, M.H. Teng, B. R. Elliott, J.-H. Hwang, D. L. Johnson, T. O. Mason, and J. R. Weertman, Nature (London) 374, 602 (1995).

31. V. P. Dravid, M.H. Teng, J. J. Host, B. R. Elliott, D. L. Johnson, T. O. Mason, J. R. Weertman, J.-H. Hwang, US Patent 5472749 (1995).

32. J. J. Host, M.H. Teng, B. R. Elliott, J.-H. Hwang, T. O. Mason, D. L. Johnson, and V.P. Dravid, J. Mater. Res. 12 (5) (1997, in press).
33. M. A. Omar, in Elementary Solid State Physics (Addison-Wesley Publishing Company, Reading, MA, 1975), p. 423.

34. Y.-W. Du, M.-X. Xu, J. Wu, Y.-B. Shi, and H.-X. Lu, J. Appl. Phys. 70 (10) 5903 (1991).

35. K. Tamura and H. Endo, Phys. Lett. 29A (2), 52 (1969).

36. W. Wagner, A. Wiedenmann, W. Petry, A. Geibel, and H. Gleiter, J. Mater. Res. 6, 2305 (1991).

37. H.-E. Schaefer, H. Kisker, H. Kronmuller, and R. Wurschum, Nanostructured Mater. 1, 523 (1992).

38. X. K. Wang, R. P. H. Chang, A. Patashinski, and J.B. Ketterson, J. Mater. Res. 9, 1578 (1994).

39. S. Ohnuma and A. Kunimoto, J. Appl. Phys. 63 (8), 4243 (1988).

40. W. Gong, H. Li, Z. Zhao, and J. Chen, J. Appl. Phys. 69 (8), 5119 (1991).

41. M. J. Aus, B. Szunar, A.M. El-Sherik, U. Erb, G. Palumbo, and K. T. Aust, Scripta Metall. et Mater. 27 (11), 1639 (1992).

42. S. A. Sethi and A. Tholen, in Mechanical Properties and Deformation Behavior of Materials Having Ultra-Fine Microstructures, edited by M. Nastasi et al. (Kluwer Academic Publishers, The Netherlands, 1993), p. 157.

43. Y. Sakka and T. Uchikoshi, Powder Metall. 36 (3), 179 (1993). 